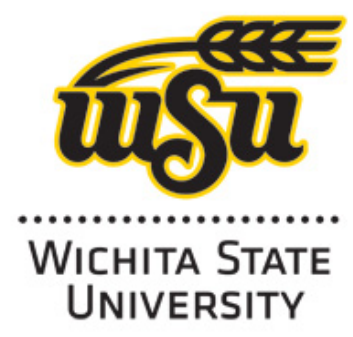

Wichita State University Libraries SOAR: Shocker Open Access Repository

\title{
SVSS: Intelligent Video Surveillance System for Aircraft
}

Naagaraja Thanthry

Indira P. Emmuadi

Aravind Srikumar

Kamesh Namuduri

Ravi Pendse

\section{Affiliation: \\ Naagaraja Thanthry, Indira P. Emmuadi, Aravind Srikumar, Kamesh Namuduri \& Ravi Pendse, Department of Electrical and Computer Engineering, Wichita State University, Wichita, KS.}

\section{Recommended citation}

Thanthry, Naagaraja., Emmuadi, Indira P., Srikumar, Aravind., Namuduri, Kamesh and Ravi Pendse. 2009. SVSS: Intelligent Video Surveillance System for Aircraft. IEEE Aerospace and Electronic Systems Magazine, Volume 24 , Issue 10, pp. 23-29. DOI 10.1109/MAES.2009.5317783 


\section{SVSS: Intelligent Video Surveillance System for Aircraft}

Naagaraja Thanthry, Indira P. Emmadi, Aravind Srikumar, Kamesh Namuduri \& Ravi Pendse Wichita State University

\begin{abstract}
Safety and security are the most discussed topics in the aviation field. The latest security initiatives in the field of aviation propose [I] the aircraft carriers to implement video surveillance within the aircraft at strategic locations. The current proposals allow the video surveillance data to be stored within the aircraft and monitored by one of the flight crew. The monitoring crew will be responsible for identifying the anomaly within the aircraft and take necessary preventive actions. With the introduction of additional technology within the aircraft, mere human perception may not be sufficient to make a decision. In this research work, the authors explore the possibility of implementing a smart video surveillance system (SVSS) within the aircraft that is tuned toward detecting the behavioral anomaly within the aircraft. The SVSS will generate security triggers when it detects an anomaly within the aircraft. These triggers could be combined with other triggers generated by different aircraft components (possible alarms from the flight crew, data traffic anomaly, or alarm generated by one of the avionics components) to provide a better understanding of the situation to the monitoring crew.
\end{abstract}

\section{INTRODUCTION}

Safety is one of the major aspects in today's aviation world. A safe take-off or a safe landing does not ensure the complete safety and security of the flight. Passenger and crew safety when the aircraft is in flight requires a better surveillance system within the aircraft. This calls for a better design, both in terms of placement of the surveillance devices, as well as the technique.

A peephole on the cockpit door is one of the cost-effective solutions that is implemented in many of the aircrafts. The peephole is not the effective solution as it strains the flight crew both physically and mentally. Some countries have implemented cabin video-surveillance systems which require pilot's involvement in their operation as there is no intelligence built in them. Moreover, after 20 minutes of surveillance, in all such non-automated vigilance systems, human attention to the video details degenerate into an unacceptable level and the video surveillance becomes meaningless. Thus there is an increasing demand for intelligent video surveillance systems with automated tracking and alerting mechanisms [2].

Developing such a system to monitor and authenticate the person requesting entry into the cockpit has multifarious challenges. Some of which are selecting appropriate face recognition algorithms, placing the cameras at strategic locations, developing an overall architecture that meets FAA regulations.

Currently, there are some off-the-shelf video surveillance systems available that could be used within an aircraft. Airworks Cockpit Door Surveillance Systems use 16 cameras and a flight deck mounted touch-screen video monitor, providing intuitive crew controls for pilot and co-pilot systems operation [I]. Goodrich video surveillance system, which initially used 16 IR cameras, have reduced the complexity by combining with an electronic flight bag (EFB), reducing the number of cameras used to four. There are several other similar implementations with no built-in intelligence (AD Aerospace FlightVu, CabinVu, Cabin Vu-123, Rockwell Collins Wireless Cabin Video Surveillance Systems).

Herein these authors propose an architecture of an intelligent video surveillance system called Smart Video Surveillance System (SVSS). These authors have evaluated three different face recognition algorithms (Principle Component Analysis [3], Linear Discriminant Analysis [4] and Trace Transforms [5] algorithms) in the Face Recognition Algorithm section. The core architecture of the 
SVSS is described in the SVSS Architecture section, followed by its working principle in the SVSS Working section. In the FAA Regulations section, these authors show that the proposed architecture meets all the requirements of FAA policies and regulations [6], dated October 5, 2001. Finally, the authors conclude the paper with the highlights of the SVSS and with a scope for further research.

\section{FACE RECOGNITION ALGORITHMS}

Image-based Face Recognition involves both the representation and the implementation details of the standard recognition of the images and Face recognition considering the Face Perception is still an on-going research area. Herein these authors analyzed the following face recognition algorithms and suggested the usage of PCA-LDA and Trace Transform.

\section{Principle Component Analysis (PCA)}

Linear space techniques, such as PCA and LDA, are used for reducing dimensionality and for feature extraction. In PCA, the face is treated as a two-dimensional vector space or face space. The face space is defined as the collection of Eigen vectors of a set of faces. In this most popular appearance-based approach, the face images are transformed into a small set of characteristic feature images called Eigen faces which are the principle components of the initial set of images [7]. Recognition is performed by projecting a new image to the sub-space spanned by Eigen faces and classifying the faces by comparing their position in the face space with the known faces.

\section{Eigen Face Approach}

- From the image set (training set) calculate the Eigen faces for the set of images that correspond to the highest Eigen values. This forms the face space.

- Update the face space with the addition of a new face image and form the face space.

$$
P C A=R n \text { Image }=R m
$$

The method involves finding an $\mathrm{n}$ dimensional sub-space with basic vectors that correspond to minimal variance in the vector space with dimension $\mathrm{m}$. So it reduces the dimensionality and complexity in computation $(m>n)$.

\section{Linear Discriininant Analysis (LDA)}

Eigen faces method is one of the most successful methods which use the PCA algorithm for feature extraction, and is done by finding the optimal projection direction that preserves the data variance to its maximum. But PCA does not take into account the class label information and the optional projection direction [8]. Even though it is useful for data reconstruction and representation it loses the quality of discrimination, so it is not appropriate to use PCA to discriminate between different classes of images. LDA seeks the optimal projection direction to minimize scattering between classes to within class scattering [9]. LDA uses either weighted or un-weighted distances.

When the number of training samples per class is large, LDA is the best choice. Discriminant analysis of Eigen features is applied to determine not only class (human face vs. non-face objects) but also individuals within the face class. Using tree-structure learning, the eigenspace and LDA projections are recursively applied to smaller and smaller sets of samples. Such recursive partitioning is carried out for every node until the samples assigned to the node belong to a single class.

Since the image or face space dimension is very high compared to the number of images in the training set, it creates computational difficulty in LDA algorithms, i.e., the within class scattering matrix is singular, so LDA alone cannot be applied. This problem can be attributed to under-sampling of data in high dimensional image space. A comparative performance analysis was carried out by Belhurneur et al. suggested that this problem can be avoided by applying PCA first and then followed by LDA in fisher-faces [10]. Past research has shown that fisher-face methods give better results than the Eigen-face method in various cases.

We know that the fisher-face is superior to the Eigen-face approach when the training images contain much variation in an individual class; otherwise the performances of fisher-face and Eigen-face are not significantly different [I I]. Some principle components with small Eigen values can correspond to high frequency components which can produce noise in fisher-face [12]. To avoid this drawback, later enhanced LDA methods were proposed for face recognition which simultaneously digitalize the within class scatter and between class scatter matrices of conventional LDA procedures. Zhao et al. proposed a way to unite PCA and LDA with the use of regularized sub-space LDA.

The hypothesis and the architecture of the video surveillance system put forward by these authors are based on a small set of images. So, these authors use both PCA and LDA for face recognition. These authors define the accuracy by setting the threshold for each of the image-based face recognition algorithms.

\section{Trace Trans form}

Trace transform is calculated by calculating functional values (i.e., any function, for example $T[f(t)]=O)^{\infty} f(t) . d t$ ) along the tracing lines of the image as shown in Figure 1. Similarly, other functionals along image lines can also be computed. By doing so we found that the functional representation of the image does not vary with scaling, illumination, or rotation which illustrates the discriminating ability of trace transform [13]. By employing appropriate 


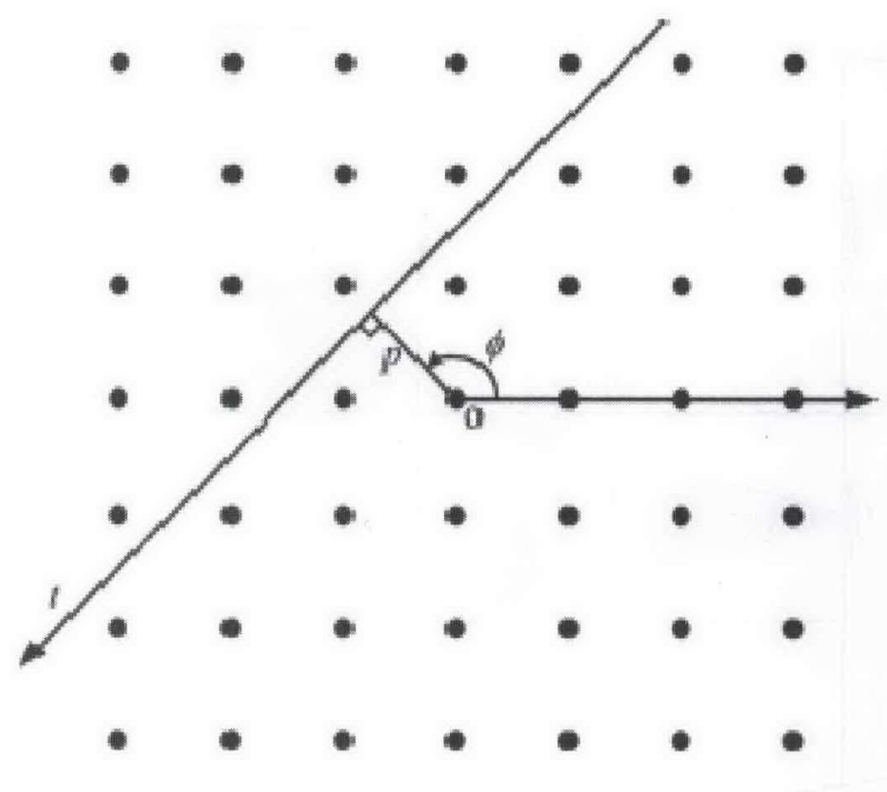

Fig. 1. Tracing Line Illustrated Along with Parameters $\Phi_{2}, \mathbf{t}$ and $\mathbf{p}$ [5]

Table 1. Trace Functionals [5]

\begin{tabular}{|l|l|l|}
\hline$N_{0 .}$ & \multicolumn{1}{|c|}{ Trace Functionals } & Details \\
\hline 1 & $T(f(t))=\int_{0}^{\infty} f(t) d t$ & \\
\hline 2 & $T(f(t))=\int_{c}^{\infty} \sqrt{|t-c|} f(t) d t$ & $c=\operatorname{med}\{t, f(t)\}$ \\
\hline 3 & $T(f(t))=\int_{c}^{\infty}(t-c)^{2} f(t) d t$ & $\begin{array}{l}c=\frac{1}{S} \int_{0}^{\infty} t|f(t)| d t, \\
S=\int_{0}^{\infty}|f(t)| d t\end{array}$ \\
\hline 4 & $T(f(t))=\left[\int_{0}^{\infty}|f(t)|^{p} d t\right]^{q}$ & $p=0.5, q=1 / p$ \\
\hline
\end{tabular}

training techniques, trace transform can be used to reduce inter-class variance and intra-class variance. Trace transform may be used to address practical difficulties in face authentication, recognizing a face image with different viewing directions or presence of illumination. With appropriate functional trace transform can be used for face authentication.

Linear space techniques (sub-space face recognition algorithms) work accurately based on the efficiency of the face feature extraction. Unlike linear space techniques, in trace transform, face feature representation, depending upon the choice of the functional used, increases the discriminating ability of the face authenticating framework. Table 1 shows a few examples of functionals most commonly used.

To calculate the trace transform and to make the system robust to shape changes in the images, the face image should be extracted within a well-defined structure such as an ellipse or a circle. The extracted image is trace transformed to another image with the trace transform functional which is a two-dimensional function of variables $\Phi$ and $p$ in the Cartesian coordinate system as shown in the Figure 1.

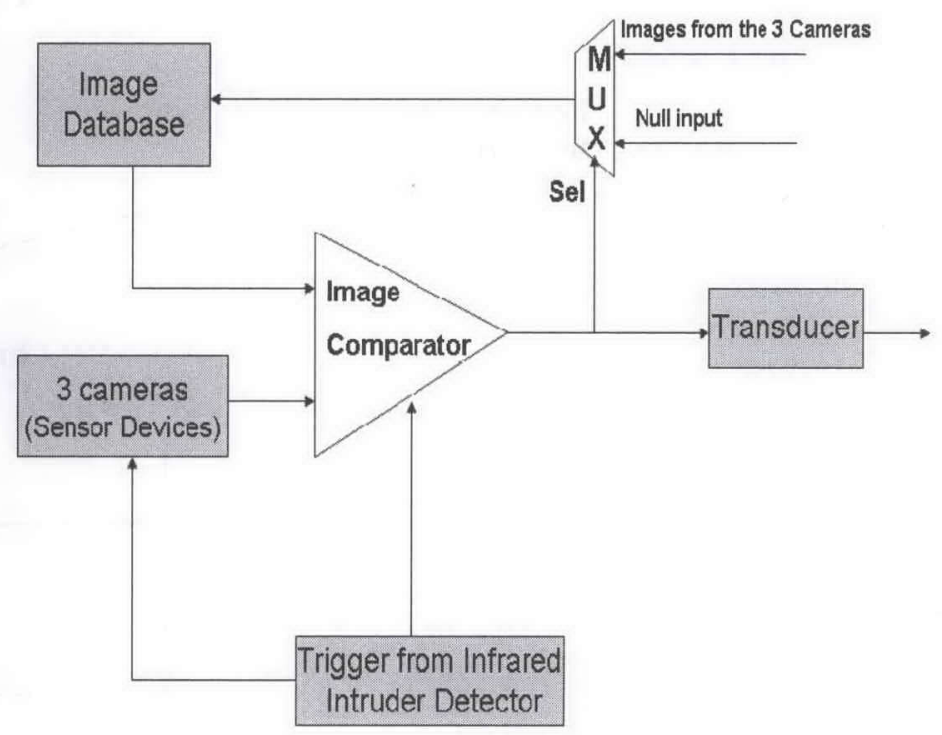

Fig. 2. Block Diagram of the Control System

Past research has shown that trace transform has a higher recognition rate than the Eigen-faces method in all cases [5]. These authors preferred to use trace transform algorithm (with a threshold set to define the accuracy) as the second face recognition algorithm.

\section{SVSS ARCHITECTURE}

The advantages of an intelligent surveillance system outweigh the challenges of a smart surveillance system. For an automatic authentication, SVSS uses an Image database, Cameras, Infrared sensors, an Image Comparator, Feedback selection (Multiplexer) system, and an audio-visual alerting system for the flight crew. Figure 2 shows the block diagram of the control system.

\section{Database Server}

The database contains the pre-stored images of the flight crew which is stored in the database server. This server also stores the images from the cameras which require further monitoring. Databases are classified into various image galleries depending upon the computational time of specific algorithms. For instance, the computational time of the PCA 


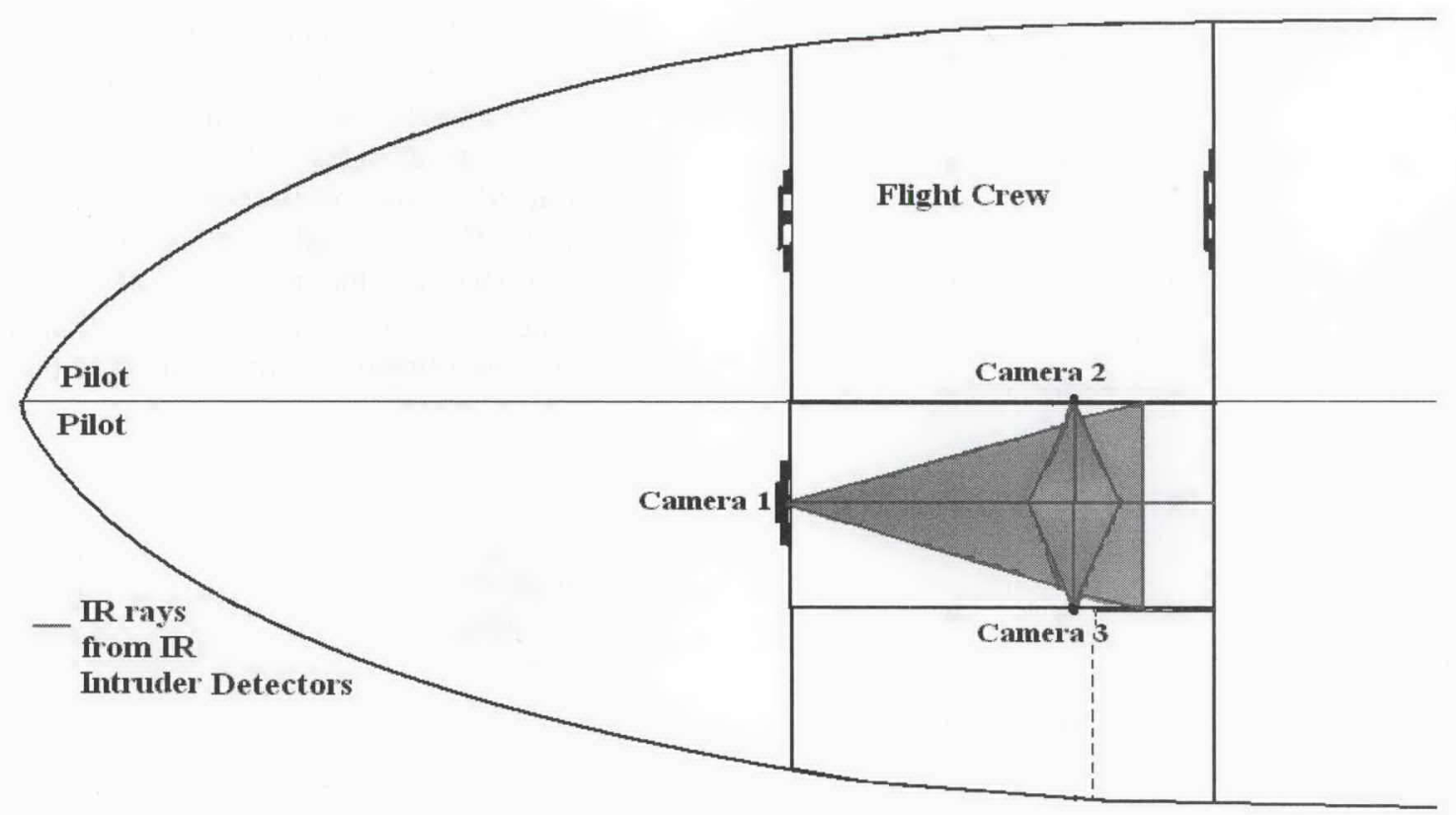

Figure 3(a)

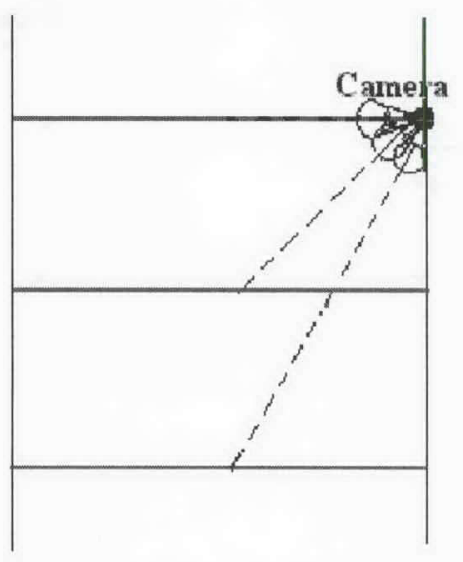

Fig. 3A. Vertical View Showing the cameras placed in the cockpit passage;

Fig. 3B. Horizontal View of a beam of rays that are viewed as a single ray in Figure 3A

algorithm depends on the type of distance measure used as a classifier or training set. (Input image should be in gray-scale and pre-normalized.)

\section{Sensor}

A simple Infrared intrusion detection system is used to sense the intrusion and activate the surveillance system. It is employed in time-scaling the images by the cameras.

\section{Cameras}

We use three high-resolution digital cameras, which are placed in the cockpit passage to capture the images of the person requesting an entry into the cockpit. The cameras are strategically placed as shown in Figure 3A.

\section{Image Comparator}

The actual part of the face recognition takes place here. The comparator comprises two application servers each running a separate image-based face recognition algorithm. The servers compare the input image with the images in the database server that fall with in the same class or with the images for which the distance is small. These application servers convert the output of the implemented face recognition algorithms into a binary form.

\section{Selection (MUX) System}

This server comprises simple multiplexer code with two selection lines and decides whether the image should be added to the database for further monitoring, depending on the output of the image comparator.

\section{Transducer}

The transducer is used to convert the digital output from the image comparator into an audio visual which alerts the pilot. 


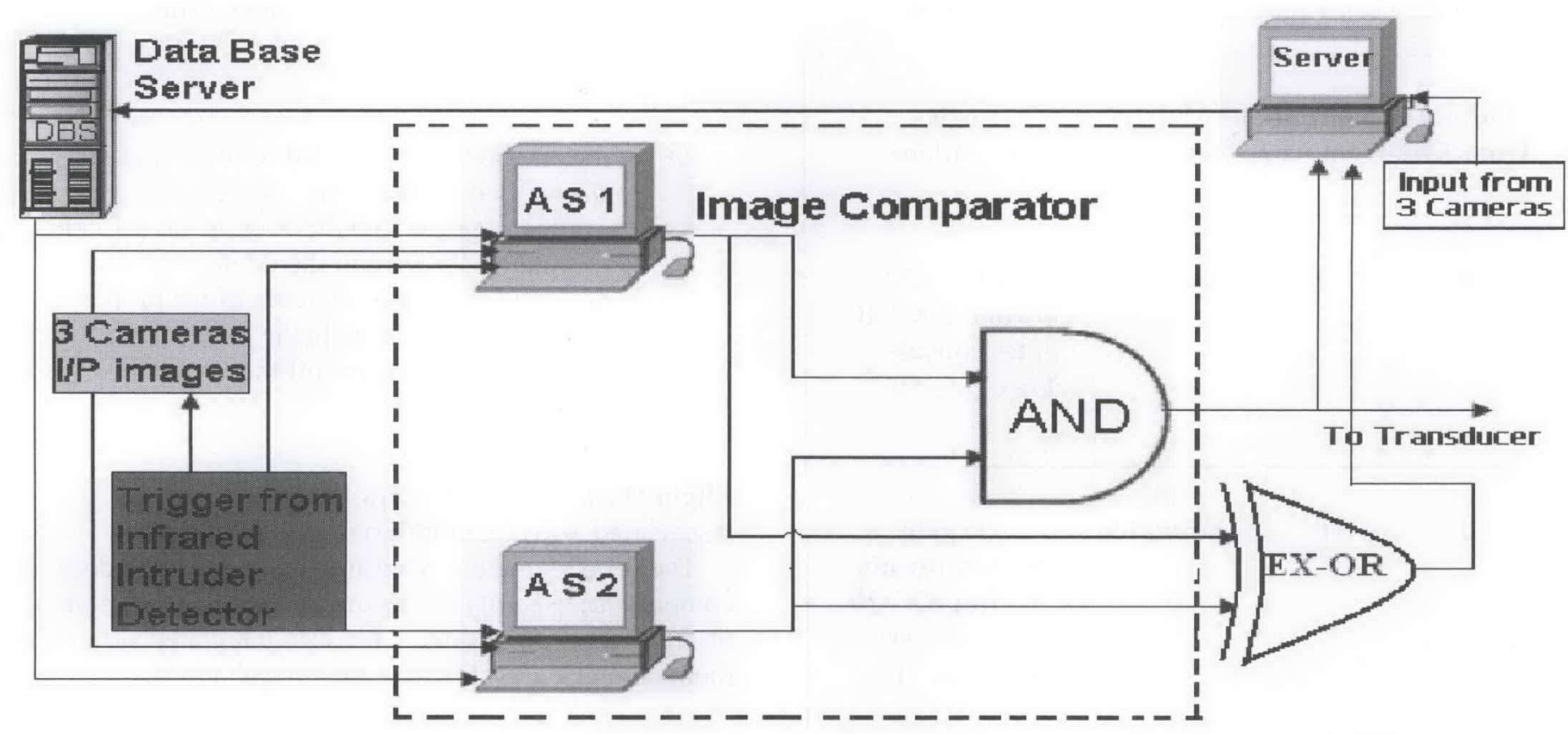

Fig. 4. Comparator Diagram

\section{SVSS WORKING}

When a person tries to access the cockpit door, the high resolution cameras are triggered with input from efficient IR Intruder detectors that use infrared rays. The three strategically placed cameras set their angles according to the height of the intruder which is, in turn, determined by the IR rays as shown in Figure 3B, and take his/her images.

The intelligence of the system is built in the image comparator, which consists of two high performance application servers, each running an efficient image-based face recognition algorithm. These algorithms compare the input image train with the images of flight crew members stored in the database server.

Figure 4 illustrates the functional block diagram of the image comparator. Each algorithm has its own independent thresholds set, defining the accuracy levels, thus confirming the match. Based on the distance from the threshold, the output of the algorithm is programmed to be a binary signal ( 0 or 1 ). Accordingly, there will be four possible output combinations from server 1 and server 2 , which are later logically operated as shown in Table 2 . The Logic operations reduce the probability of error in the image comparator. The control system of the SVSS is designed with an implicit Deny action.

If there is a match then the person is allowed to enter the cockpit only upon the pilot's approval. If there is a Complete No Match (i.e., the output combination of the application servers being 00 ), then the flight crew will be alerted. Moreover, these low outputs of both the AND and EX-OR gates select the No-Match image at the Multiplexer and store it in a different logical location of the database for further monitoring and future occurrences.
When the output combination of the application servers is 01 or 10, the EX-OR gate produces a high output and eventually selects the input image at the multiplexer and stores it in a different logical location on the database server. An alert signal is sent to the pilot to analyze these images if they are any chances for a match.

While the proposed system could function all by itself in recognizing and authorizing personnel, it is not intended to override the authority of the flight crew within the aircraft. The proposed system will assist the flight crew in making a decision when the need arises. In addition, the proposed design could be used as part of an automated flight control system which, in stress situations, could trigger download of critical parameters (including surveillance video) to the ground-station for further analysis. This improves flight safety and also allows the regulatory authority to have a better insight of the proceedings within the aircraft.

\section{FAA REGULATIONS}

Any surveillance systems to be implemented should meet FAA requirements. The following policies and specifications are the necessary requirements that are to he met to ensure a standardized approach in any surveillance systems within the aircraft [5].

\section{Electrical and Installation Issues}

- In accordance with $\S 25.1301$, the Cabin Mounted Video Cameras (CMVC) system must be of a kind and design appropriate to its intended function, and must function properly when installed. (The whole goal is to design an 
Table 2. Output of the Image Comparator

\begin{tabular}{|c|c|c|c|}
\hline $\begin{array}{l}\text { Output } \\
\text { from AS1 }\end{array}$ & $\begin{array}{c}\text { Output } \\
\text { from AS2 }\end{array}$ & $\begin{array}{c}\text { Match / } \\
\text { No Match }\end{array}$ & $\begin{array}{c}\text { Logic } \\
\text { Operations }\end{array}$ \\
\hline 0 & 0 & No Match & $\begin{array}{l}\text { Both the AND } \\
\text { gate and EX-OR } \\
\text { gates consider } \\
\text { the o/p as "0" }\end{array}$ \\
\hline 0 & 1 & No Match & $\begin{array}{c}\text { AND gate } \\
\text { considers the o/p } \\
\text { as " } 0 \text { " But EX-OR } \\
\text { gate considers } \\
\text { the } 0 / p \text { as " } 1 \text { " }\end{array}$ \\
\hline 1 & 0 & No Match & $\begin{array}{c}\text { AND gate } \\
\text { considers the o/p } \\
\text { as "0" But EX-OR } \\
\text { gate considers } \\
\text { the o/p as "1" }\end{array}$ \\
\hline 1 & 1 & Match & $\begin{array}{l}\text { AND gate } \\
\text { considers the o/p } \\
\text { as "1" But EX-OR } \\
\text { gate considers } \\
\text { the o/p as "0" }\end{array}$ \\
\hline
\end{tabular}

effective surveillance system (i.e., SVSS) with appropriate and sufficient built-in intelligence).

- The CMVC system components and wiring should meet the flammability requirements of $\S$ 25.853 and $\S 25.869$. Wiring should be protected by appropriately rated and coordinated circuit breakers in accordance with $\S 25.1357$ and the system components should be installed in accordance with wiring standards established by the original aircraft manufacturing.

- System separation and required instrument systems should not be compromised by the installation of the CMVC system. (We will be using a stand-alone system which will not interfere with the existing system).

- The CMVC system should be connected to an electrical bus that does not supply power to airplane systems that are necessary for continued safe flight and landing. (This proposed control system powered with a lower level of electrical busses).

- A means should be provided for the flight crew to manually disconnect the CMVC system from its source of power and the only means provided to disconnect the system should not be circuit breakers. (The control switches of the proposed system should be placed in the pilots cabin allowing the pilot to turn-off the system if deemed necessary).

\section{Flight Deck Human Factors Issues}

Associated with Installation of CMVC Systems

The primary issue is to ensure that installation does not compromise usability of existing systems. Stand-alone-type of installation is considered because Integrated systems are more complex and critical in their installations.

\section{Issues Associated With Stand-Alone Installations}

- $\$ 25.773(a)(2)$ Ensure that the video display unit does not produce unacceptable glare or reflections on the existing essential/critical displays or on the flight deck windows, under all expected lighting conditions. (We are using IR motion/intruder detectors to power on the control system only when needed).

- $\$ 25.777$ (a) Ensure that the installation does not place the controls for the camera system in locations that may result in inadvertent operation of other controls. (The system is architectured with built-in intelligence so that strain of the flight crew is reduced).

- $\$ 25.1523$ The use of the system, in accordance with the proposed operating procedures, should not result in pilot distraction or workload that may unacceptably compromise pilot performance of other required tasks. (Pilot involvement in the control system is minimized with built-in intelligence in the proposed system).

Henceforth, the proposed system meets all FAA requirements.

\section{Advantages of SVSS}

The following are the disadvantages of the present non-intelligent video surveillance systems such as CMVC:

- The cabin crew is responsible for monitoring and detecting in-flight anomalies. 
- Function is similar to a look-through hole in the cockpit door.

- Surveillance is less efficient.

- Stress on flight crew is high.

The highlights of the proposed SVSS, which overcomes most of the limitations of the existing non intelligent CMVC, are listed below:

- Authenticates the person requesting access to the cockpit automatically (reduces the load on the pilot).

- Makes the surveillance more efficient.

- Alerts the cabin crew in case of anomalies.

- Increases flight crew and passenger safety.

- Reduce the waste of power and unnecessary video surveillance in cases when not required with the integration of Infrared intruder detector in the SVSS.

\section{CONCLUSIONS}

Herein, these authors discussed the need of an intelligent surveillance system within the aircraft and proposed architecture for same. The advantages of an intelligent surveillance system outweigh the challenges of a smart surveillance system. The complexity and efficiency of the control system depend solely on the functional accuracy of the image-based face recognition algorithms. These authors propose using a hybrid image recognition algorithm that takes into account both the face dimensions as well as features. These authors reviewed the FAA requirements for a surveillance system to be installed within the aircraft and proved that the proposed architecture meets all requirements.

As a future work, these authors intend to develop a hybrid image recognition algorithm that can recognize images with highest accuracy. In addition, these authors also plan to build a prototype of the proposed architecture and evaluate its performance under various conditions.

\section{REFERENCES}

[1] Heather Baldwin, June 2004, Securing Airline cabins, Homeland Security.
[2] Case Study by Seagate,

Smart Surveillance Systems Tap Reliability and Performance of Seagate SV35 Series Drives.

[3] W. Zhao, R. Chellappa and A. Krishnaswamy, 1998, Discriminant analysis of principal components for face recognition, In $3^{\text {rd }}$ International Conference on Automatic Face and Gesture recognition.

[4] Guang Dai, Dit Yan Yeung and Yun Tao Qian, 2007, Face Recognition using Kernel-step discriminant analysis algorithm, Pattern Recognition, 40, (2007), 229-243.

[5] Alexander Kadyrov and Maria Petrou, 2001, The Trace Transform and Its Applications, IEEE Computer Society Washington, DC, USA.

[6] INFORMATION: Interim Summary of Policy and Advisory Material Available for Use In the Certification of Cabin Mounted Video Cameras Systems with Flight Deck Displays on Title 14 CFR,

Part 25, Aircraft Date: October 5, 2001.

[7] Matthew Trunk and Alex Pentland, 1991, Eigenfaces for Recognition, Journal of Cognitive Neuroscience, Vol. 3, No. 1, pp. 71-86.

[8] Kresimir Delac, Mislay Grgic and Sonja Grgic, FER. Unska 3/XI i, Zagreb, Croatia February 2006, Independent Comparative Study of PCA, ICA, and LDA on the FERET Data Set, University of Zagreb.

[9] Guano Dai, Dit Yan Yeung and Yun Tao Qian, 2007, Face Recognition using Kernel-step Discriminant analysis algorithm, Pattern Recognition, 40, pp. 229-243.

[10] P.N. Belhumeur, J.P. Hespanha and D.J. Kriegman, May 1997, Eigenfaces vs. Fisherfaces: Recognition using class specific linear projection, IEEE Trans. Pattern Anal. Machine Intell., Vol.19, pp. 711-720.

[11] C. Liu and H. Wechsler, April 2001, A Shape- and Texture-Based Enhanced Fisher Classifier for Face Recognition, IEEE Transactions on Image Processing, Vol. 10, No. 4, pp. 598-608.

[12] W. Zhao, R. Chellappa, J. Phillips and A. Rosenfeld, December 2003, Face Recognition in Still and Video Images: A Literature Survey, ACM Computing Surveys, Vo1. 35, pp. 399-458.

[13] Sanun Srisuk, Kongnat Ratanarangsank, Werasak Kurutach and Sahatsawat Waraklang, Face Recognition using a New Texture Representation of Face Images. 